\title{
Distributed Bio-Hydrogen Refueling Stations
}

\author{
Peter J. Schubert \\ Richard G. Lugar Center for Renewable Energy, Indiana University-Purdue University Indianapolis, Indianapolis, IN, 46202, \\ United States
}

\begin{abstract}
Hydrogen fuel cell cars are now available for lease and for sale. Renewable hydrogen fuel can be produced from water via electrolysis, or from biomass via gasification. Electrolysis is power-hungry with high demand from solar or wind power. Gasification, however, can be energy self-sufficient using a recently-patented thermochemical conversion technology known as I-HPG (indirectly-heated pyrolytic gasification). I-HPG produces a tar-free syngas from non-food woody biomass. This means the balance of plant can be small, so the overall system is economical at modest sizes. This makes it possible to produce renewable hydrogen from local agricultural residues; sufficient to create distributed refueling stations wherever there is feedstock. This work describes the specifics of a novel bio-hydrogen refueling station whereby the syngas produced has much of the hydrogen extracted with the remainder powering a generator to provide the electric power to the I-HPG system. Thus the system runs continuously. When paired with another new technology, moderate-pressure storage of hydrogen in porous silicon, there is the potential to also power the refueling operation. Such systems can be operated independently. It is even possible to design an energy self-sufficient farm where all electric power, heat, and hydrogen fuel is produced from the non-food residues of agricultural operations. No water is required, and the carbon footprint is negative, or at least neutral.
\end{abstract}

Key words: Biomass, hydrogen, distributed generation, fuel cell vehicle.

\section{Introduction}

Hydrogen is an energy vector, not a source. Hydrogen is bound in molecules and must be extracted. Once extracted, hydrogen can be stored or transported for later use, or converted immediately to heat, electricity or mechanical work. Each transition incurs an energy penalty. Transitions and associated equipment/facilities furthermore incur environmental costs. In seeking sustainable solutions parasitic energy and effluents must be considered. Most global hydrogen production involves steam reforming of methane from natural gas, an endothermic process which releases fossil-sourced carbon dioxide. Releases of $\mathrm{CO}_{2}$ and leaks of fugitive $\mathrm{CH}_{4}$ become greenhouse gases, which are much to be avoided. An alternative is solar hydrogen where photovoltaics provide the power to electrolyze water into $\mathrm{H}_{2}$ and $\mathrm{O}_{2}$. Electrolysis is energy-intensive, hence costly. A superior alternative is extraction from

Corresponding author: Peter J. Schubert, Ph.D., P.E., professor of electrical and computer engineering, director, research fields: renewable energy generation, transmission, and storage. E-mail: pjschube@iupui.edu. high-hydrogen producer gas made by a novel form of biomass gasification called I-HPG (indirectly-heated pyrolytic gasification) [1-3]. High purity hydrogen slipstreams can be extracted from the producer gas (a.k.a. "syngas") via membranes, with the remaining gases used in a fuel cell or internal combustion engine to provide electric power to sustain operations.

Commercial methods of hydrogen storage are cryogenic, high pressure, and solid state metal hydrides. Parasitic energy requirements for cooling to below $20^{\circ} \mathrm{K}$, or for pressurizing the 700 bar tanks in current hydrogen FCVs (fuel cell vehicles) are considerable. Metal hydrides have low specific capacity, and are highly exothermic upon recharge, making systems bulky and complex. A new method of solid state hydrogen storage is catalytically-modified porous silicon [4-7]. Strategically placed catalyst atoms facilitate separation of the $\mathrm{H}-\mathrm{H}$ bond, allowing spillover of hydrogen atoms onto the porous silicon surface $\left(800 \mathrm{~m}^{2} / \mathrm{gm}\right)[8,9]$. Recharge pressures as low as 2.5 bar are possible [10]. Furthermore, silicon is earth abundant and environmentally benign. 
This combination of bio-hydrogen with storage in porous silicon opens up a new paradigm for FCV refueling stations [11]. Instead of central plants and pipelines we have farmers bringing non-food biomass to distributed facilities along roadways. Instead of power-hungry compressors and chillers we have small mechanical pumps. As is shown in this paper, distributed I-HPG bio-hydrogen can deliver fuel to a porous silicon storage vessel at on-going costs which are below the current market rate [12]. The transformative potential of this new paradigm can hardly be overstated.

\section{Methods}

The operation of I-HPG is to indirectly heat lignocelluosic material in the absence of air gases to a temperature of $950{ }^{\circ} \mathrm{C}$. At this temperature all carbon-based compounds break down into their simplest components, either $\mathrm{H}_{2}, \mathrm{CO}$, or pure carbon char (a.k.a. "biochar"). This producer gas (formally) or syngas (colloquially) is inherently low in tars or heavy hydrocarbons which are the bane of conventional gasifiers. Entrained particles are removed downstream of the plug-flow, indirectly-heated tubular reactor via a small cyclone, and then a sintered metal filter. After cooling via heat exchangers water vapor is removed by condensation and de-watering apparatuses. A detailed thermodynamic model was created based on elemental analysis of wood chips [13] with 20 percent moisture content by weight. Measured syngas from I-HPG indicates $45 \% \mathrm{CO}$ and $45 \% \mathrm{H}_{2}$. Subtracting heats of formation from 5-term wide range heat capacity [14] and balancing by element yields a gas composition of $51.8 \% \mathrm{CO}$ and $47.8 \% \mathrm{H}_{2}$. This model predicts energy content of (301 BTU/cu.ft.) which is slightly lower than measured syngas (315 BTU/cu.ft.). Two key differences are the overestimate of hydrogen in the model and the underestimate of methane (4\%), however the result is considered sufficiently accurate to explore the extraction of a hydrogen slipstream from the syngas with the remaining gases used in a prime mover to run the system.

In outdoor testing I-HPG syngas has been demonstrated in a modified SI-ICE (spark-ignited internal combustion engine) designed for natural gas, but with increased input pressure and with spark timing advanced to 0 degree top-dead-center. The catalytic converter of the SI-ICE should be an automotive-grade 3-way model which consumes unburned $\mathrm{CO}$, because the hydrogen diffusion constant and flame speed are both so much higher than for CO. A single-reactor I-HPG fed at $7 \mathrm{gm} / \mathrm{s}$ produces 10.3 liters/s (24.7 SCFM) of high-hydrogen syngas according to the model. A well-tuned ICE genset with $35 \%$ electrical efficiency run on the entire gas stream will have a gross electrical output of 42 $\mathrm{kWe}$, needing $16.8 \mathrm{kWe}$ of heating power (calculated) and $5.2 \mathrm{kWe}$ of parasitic system power (measured) for a net output of $20 \mathrm{kWe}$. Theoretically a SOFC (solid oxide fuel cell) can process this blend of producer gas and achieve efficiency as high as $70 \%$ [15], and double gross output.

To produce sufficient hydrogen for a meaningful minimum number of FCV refueling events per 24 hour day the single reactor system shown in Fig. 1 is too small. A module with 6 parallel reactors can be closely packed in a thermal envelope which has less specific loss than just 1 . The mechanical components are more numerous as each tube is fed individually to maintain a plug-flow seal between the atmospheric-pressure gasification and the in-feed. A common gas collection manifold, as indicated in Fig. 2, simplifies back-end processing, somewhat offsetting the complexity penalty of the feedstock delivery system. This system can process $42 \mathrm{gm} / \mathrm{s}$ of lignocellulosic feedstock and produce an output of $286 \mathrm{kWe}$ (net of heating requirements). The total hydrogen produced is 267 $\mathrm{kg} /$ day (24 hour continuous operation). The task at hand is to determine how much of this hydrogen can be extracted by membrane while leaving sufficient chemical energy in the remaining syngas stream to provide feedstock heating, provide parasitic power, 


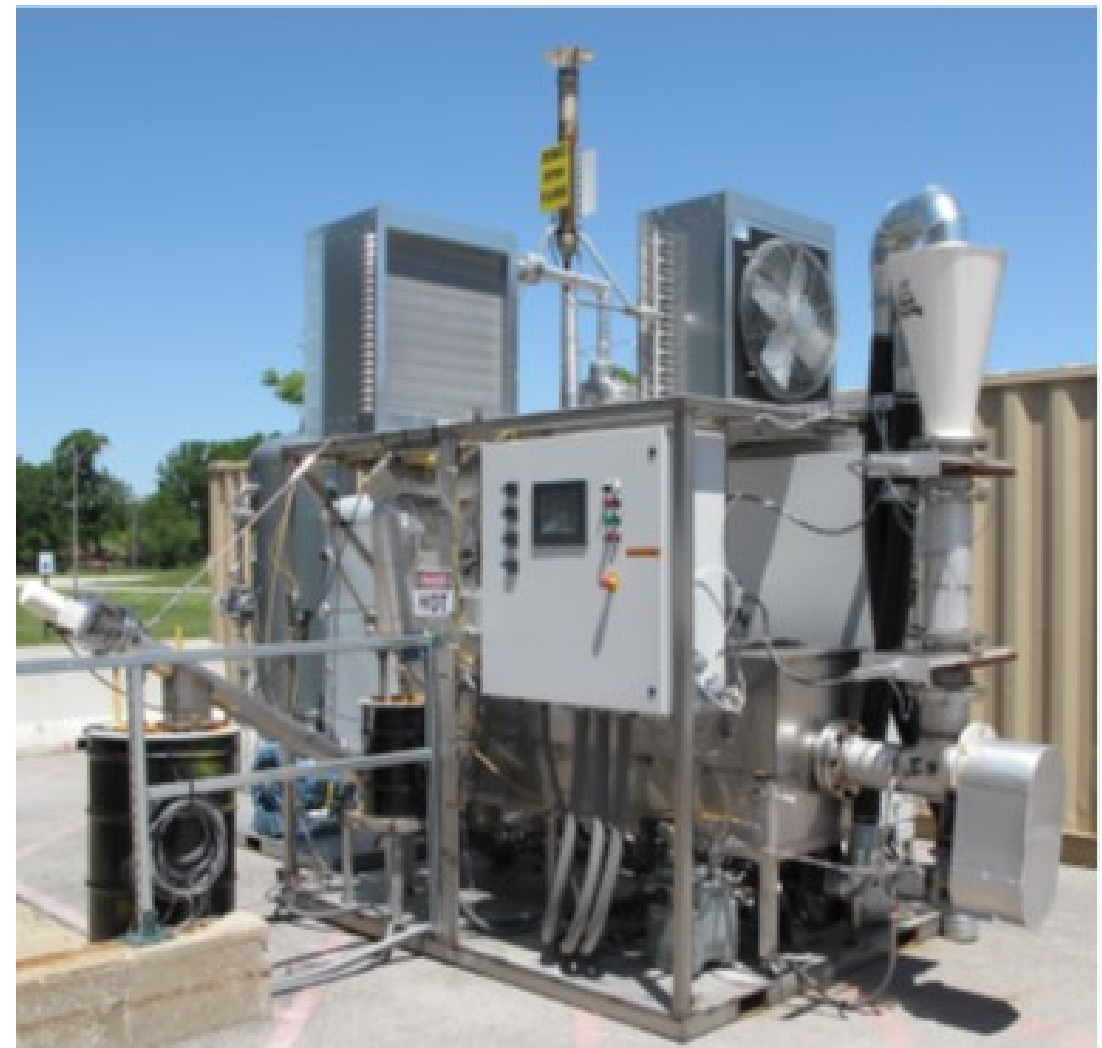

Fig. 1 I-HPFG gasifier-first pilot plant.

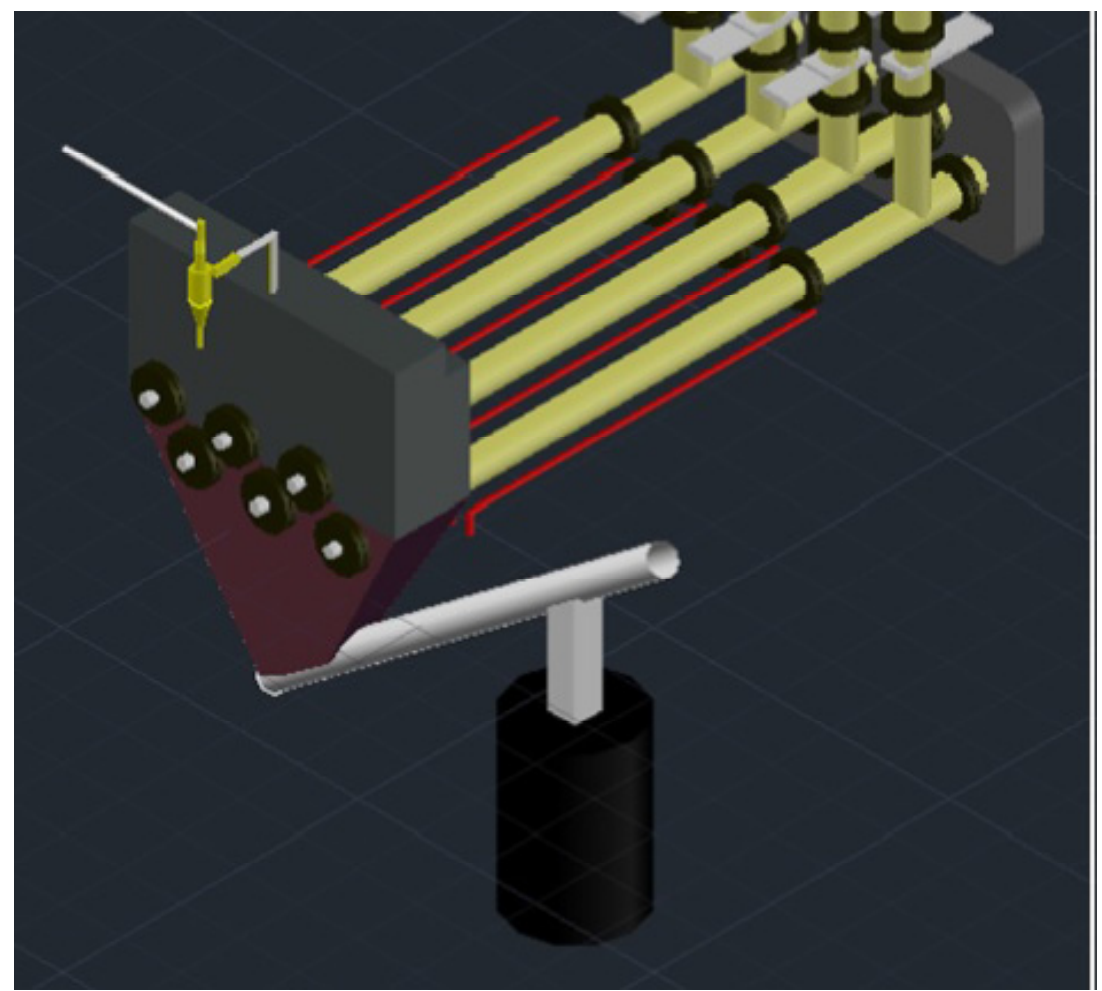

Fig. 2 I-HPFG 6-reactor module. 
and to generate the pressure needed to run the membrane compressor. In this way, so long as there is fresh biomass available to the input of the station, there will be a steady hydrogen output requiring no external input of energy.

\section{Hydrogen Separation}

The hydrogen can be separated via a heated palladium-copper membrane with a performance metric of $6.2 \mathrm{E}-5 \mathrm{~mol} / \mathrm{m}^{2}-\mathrm{s}-\mathrm{Pa}$ [16]. The work per kilogram required to pressurize an ideal gas at constant entropy is given by Eq. (1). The temperature increase for such an isentropic compression is given by Eq. (2). The ratio of heat capacities $(\mathrm{k})$ for hydrogen is 1.41 and for $\mathrm{CO}$ is 1.40 . Pressurizing by 1 bar requires $3.4 \mathrm{kWe}$, and heats the system to $440{ }^{\circ} \mathrm{C}$ which is slightly higher than the recommended separation membrane operating temperature, but thus requires no additional heating energy.

$$
\begin{gathered}
W=\frac{K R T_{1}}{k-1}\left(\left(\frac{P_{2}}{P_{1}}\right)^{\frac{k-1}{k}}-1\right) \\
T_{2}=T_{1}\left(\frac{P_{2}}{P_{1}}\right)^{\frac{k-1}{k}}
\end{gathered}
$$

Fig. 3 shows representative tube format $\mathrm{PdCu}$ membranes where thin films (approximately five microns) are deposited on refractory (alumina) tubes, then bundled with well-sealed end caps. The figure of merit for such a system is the total area of the membrane. This value was made an optimization target for the performance model subject to the constraint that the remaining gases are sufficient to all heating, parasitic, and compression power requirements.

A $\mathrm{PdCu}$ membrane of 4.7 square meters, at a 1 bar differential pressure will remove $11.0 \mathrm{~kg} / \mathrm{hr}$ from the syngas-leaving only a small percentage. The net power output is $132 \mathrm{kWe}$ over and above the internal demand, representing power which can be used to other advantageous purposes, or traded back to the utility grid for an additional revenue stream.

\section{Storage in Porous Silicon}

A high-purity slipstream of hydrogen at atmospheric pressure can be delivered directly to a waiting vehicle's fuel tank, or stored on-site for later dispensing. Both require some degree of compression. An appealing complement to bio-hydrogen from I-HPG is solid-state

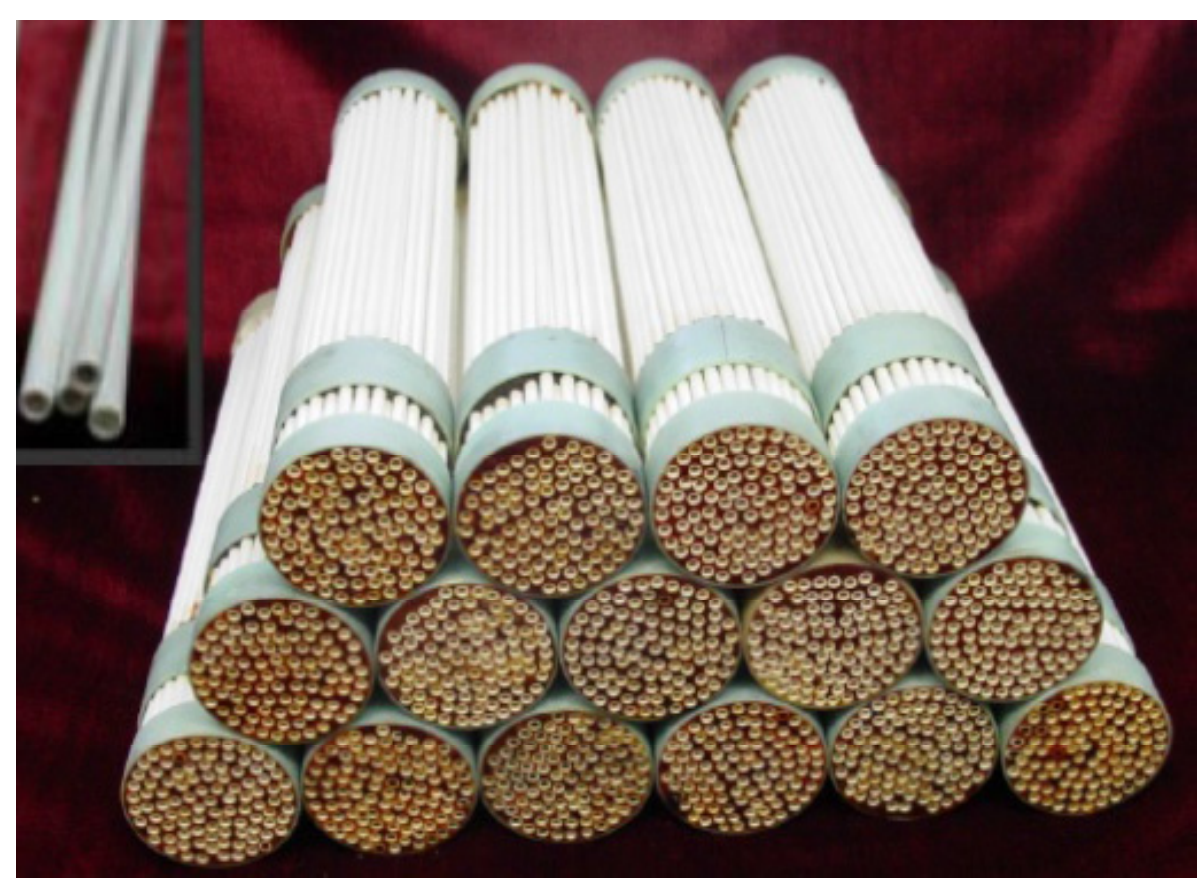

Fig. 3 Thin film Pd-Cu membranes on alumina supports for $\mathrm{H}_{2}$ separation. 
hydrogen storage using catalytically-modified porous silicon. Porous silicon is produced via electrochemical etching and forms substantially parallel columnar pores with long axes perpendicular to the surface. At certain conditions [17] these pores are uniformly 3.5 $\mathrm{nm}$ in diameter and more than 150 microns deep, exhibiting BET surface areas of $800 \mathrm{~m}^{2} / \mathrm{gm}$. When all single vacant bonds are hydrogen terminated the maximum specific storage can be as high $7.1 \% \mathrm{w} / \mathrm{w}$ [9]. Detailed energy analysis determines an overall energy change between fully-charged and fully-discharged porous silicon to be $1.2 \mathrm{~kJ} / \mathrm{mol}$ [18].

The most significant energy barrier is dissociation of gaseous dihydrogen needed for recharging this solid-state hydrogen storage medium. It is known that platinum group metals provide this catalytic function, allowing atomic hydrogen atoms which are available for transfer to the support via the spillover mechanism [18]. As-synthesized porous silicon has a FTIR signature indicating predominantly singly- and doubly-terminated surface silicon, with a small proportion triply-terminated with hydrogen-these latter having a lower bond energy. A controlled temperature excursion into the range at which hydrogen spontaneously evolves such that only these triply-terminated sites emit a pair of their hydrogen, followed by rapid quenching leaves vacant bonds strategically-placed on the remaining porous silicon matrix. Deposition of Pd(II) onto this surface creates sites where hydrogen can transfer between the gaseous state and the solid-state.

For a practical hydrogen storage medium spillover hydrogens must also bond-hop or diffuse along the silicon surface [19]. Modeled as Knudsen diffusion, the coefficient for hydrogen atoms on silicon is given by Eq. (3), where $v_{e}$ is the effective vibrational frequency, estimated as $1 \mathrm{E} 13 \mathrm{~Hz}$ [8], $z$ is the number of adjacent bond sites ("coordination number") and is taken to be $4, a$ is the nearest-neighbor distance in the silicon lattice $(0.543 \mathrm{~nm})$, and $E_{a}$ is the activation energy, which is $71.4 \mathrm{kcal} / \mathrm{mol}$ [20]. Eq. (4) shows the net diffusive flux (one dimensional) and the concentration gradient expression, found through first order discrete spatial differentiation of the ideal gas law.

$$
\begin{aligned}
& D=\frac{1}{z} v_{e} \alpha^{2} \exp \left(-\frac{E_{a}}{k T}\right) \\
& J=-D \frac{\delta n}{\delta x} \cdots \frac{\delta n}{\delta x}=\frac{P N_{A}}{R T \alpha}
\end{aligned}
$$

Conducting a numerical integration along pores of dimensions cited above yields a tradeoff between re-fill pressure and re-fill duration. When time is not a constraint, recharge times of 400 minutes (roughly "overnight") can be achieved at 2.4 bar, a pressure easily reached with an oil-free mechanical pump. For faster recharge, pressures of 8.5 bar can recharge catalytically-modified porous silicon in just 3.5 minutes. Even this higher pressure is far less than that used in pressure vessels, and as can be appreciated from Eq. (1), porous silicon requires significantly less parasitic energy for storage as a result. The faster recharge scenario requires the system to operate at $250^{\circ} \mathrm{C}$. Considering Eq. (2) this temperature is readily achieved simply by compressing the hydrogen. In fact, some cooling will be required, although significantly less than with 700 bar storage, and possibly achieved through passive means.

\section{Economic Analysis}

The cost to produce a single-reactor I-HPG system net of hydrogen separation was requested of 5 vendors, returning a mean of 240,000 USD. Current low-volume commercial costs for separating hydrogen were received at 95,000 USD, although the 2007 DOE goals suggest a mature at-volume cost of 25,000 USD may be achievable. A 6-reactor I-HPG system with hydrogen separation is, to a first order approximation, six times as costly, and so is estimated to have a cost of 1.59 MMUSD. A financial model was built which includes one-time costs for pre-processing equipment, installation, and shelter, plus on-going costs for labor, maintenance, insurance, and depreciation. Feedstock 
costs are estimated at 50 USD/MT and excess electrical generation (net of 8.5 bar $\mathrm{H}_{2}$ compression) is $0.10 \mathrm{USD} / \mathrm{kWh}$ as a cost-avoidance assuming the balance of the refueling station has a use for such. Lifetime net costs over 20 years are 4.9 MMUSD with pure hydrogen cumulative total of 1.9 million $\mathrm{kg}$ for a levelized cost of hydrogen of $2.67 \mathrm{USD} / \mathrm{kg}$.

On-site hydrogen storage at a refueling depot is needed if instantaneous demand exceeds supply. On-site storage can be modular, swappable vessels that can be exchanged in minutes; or it may be a larger multi-day store to provide reliability in the event of supply disruption. Cost estimates for a $5 \mathrm{~kg}$ storage system have been performed using derived specific costs [10] for the storage media, plus reasonable guesses for fittings, electronics, and valving. A key assumption is that porous silicon can be made from metallurgical-grade silicon instead of single-crystal silicon, although this has not yet been demonstrated in the laboratory. Module cost in this case is 5,352 USD. Scale up costs are mostly associated with the medium, and while vessel walls will scale sublinear in cost, balance of plant issues is likely to make scaling approximately linear. Thus, a 2 day on-site storage reservoir is expected to cost 565,000 USD. Adding this to the I-HPG system for capital cost and depreciation, and amortized over the expected 20 year life, the delivered cost of hydrogen is $3.26 \mathrm{USD} / \mathrm{kg}$. This is attractive relative to current bulk prices of approximately $4 \mathrm{USD} / \mathrm{kg}$, and even better compared to recent DOE Clean Cities reporting averaging 13 $\mathrm{USD} / \mathrm{kg}[21]$.

\section{Discussion}

The I-HPG technology makes economical a small-scale bioreactor suitable to distributed generation of hydrogen from local biomass. This approach side-steps the debate over who pays for a centralized hydrogen infrastructure before there is sufficient demand for it. Local bio-hydrogen refueling stations also avoid the conundrum associated with large, central biomass conversion facilities and their need to monopolize agriculture within a $80-120 \mathrm{~km}$ radius. Distributed I-HPG facilities mean that nearby farmers can bring their non-food agricultural wastes a relatively short distance and receive payment. This can be an additional cash crop. Municipalities in temperate regions can use collected landscape or utility trimmings to supply I-HPG bio-hydrogen stations. Because the operation is self-sufficient, the overall process is carbon neutral except for the embedded energy in producing the system hardware. Relative to conventional means of producing hydrogen, I-HPG bio-hydrogen comes with far less greenhouse gas emissions. Also, it requires no water and releases only steam and carbon dioxide. The mineral ash remaining after processing biomass (2-6\% typically) [13] can even be spread onto arable land to replace minor nutrients.

It is conceivable that farm operations using vehicles which run on hydrogen can power their entire operations (heat, electric power, transportation fuel) using the residues left after harvesting their cash crop (e.g. corn stover left after harvesting corn kernels). For a farm to become energy self-sufficient means that economic uncertainties are reduced, off-farm energy purchases are reduced or eliminated, and environmental footprint is reduced relative to current practices. One can also envision rural remote villages powering education, entertainment, and light industry in this way, while also providing mobility to reach more markets. In those regions of the planet where desertification is accelerating due to biomass harvesting the more-efficient use of biomass in an I-HPG gasifier can put less demand on gathered woody biomass and greater energy self-sufficiency for residents.

In a community, or nearby a facility, it is possible to have a 6-reactor I-HPG serve as a power back-up. By shutting off gas flow to the hydrogen separation membrane, the syngas can produce $291 \mathrm{kWe}$ with a $35 \%$ efficient genset, or double that with a 
high-quality fuel cell. Arranging eight "6-bangers" radially around a central feedstock hopper and metering system then provides a 2 to 4 MWe district power station. When the need for back-up power passes, the facility can return to delivering hydrogen at a cost below market rate, likely leading to steady jobs, local revenue generation, and more prosperous farmers.

Return on investment depends on the market price for hydrogen delivered to a vehicle. A reasonable range for comparison is bounded by $5 \mathrm{USD} / \mathrm{kg}$ and 8 USD $/ \mathrm{kg}$. The cost model shows simple payback before taxes of 4.8 years at the higher market price and 12.9 years at the lower price. It is also possible that some consumers may pay a premium for the benefit to the environment, support of local businesses, and energy independence.

\section{Summary}

Presented here is a novel approach to hydrogen refueling stations using non-food biomass in a manner which can become part of a distributed capability. The installed cost for each station is 2.1 MMUSD. Locally-sourced biomass provides local revenues, and I-HPG can serve to reduce solid waste streams of woody biomass, crop residue, used pallets, sawdust, utility trimmings, driftwood, and even non-halogenated plastics (presuming no reuse or recycling is available). When paired with a porous silicon solid-state hydrogen storage media the energy penalty for storage is greatly reduced relative to current practice, and the result is delivered hydrogen at the below-market rate of $3.26 \mathrm{USD} / \mathrm{kg}$. I-HPG and porous silicon can make bio-hydrogen economical and clean. This represents a new paradigm in renewable transportation fuels.

\section{Acknowledgements}

The author gratefully acknowledges Mr. Joe Paganessi who prepared the reactor models used in this work.

\section{References}

[1] Schubert, P. 2014. System and Method for Controlling Char in Biomass Reactors. US 8,691,115.

[2] Schubert, P. 2013. Scalable Biomass Reactor and Method. US 8,465,562.

[3] Schubert, P., and Ogborn, C. 2014. Process and System for Syngas Productionfrom Biomass Materials. US $8,845,772$.

[4] Yen, D., Zhang, J.-Y., and Schubert, P. 2010. Hydrogen Storage Tank and Method of Using. US 7,721,601.

[5] Schubert, P., and Christenson, J. 2010. Process and Apparatus for Producing Porous Materials. US $7,833,428$.

[6] Schubert, P. 2013. Solid-State Hydrogen Storage Media and Catalytic Hydrogen Recharging Thereof. US $8,518,856$.

[7] Schubert, P. 2014. System, Methods and Materials for Storing and Retrieving Hydrogen. US 8,673,811.

[8] Schubert, P., and Wilks, A. 2009. "Thermodynamic Analysis of a Novel Hydrogen Storage Material: Nanoporous Silicon." In Materials Innovations in an Emerging Hydrogen Economy, edited by G. Wicks, J. Simon. J. Wiley, Hoboken, New Jersey, 105-10.

[9] Schubert, P., and Babcock, J. 2013. "Advances in Synthesis of Porous Silicon for Hydrogen Storage." Proceedings Abstract of World Hydrogen Technologies Conference, Shanghai, China.

[10] Schubert, P., and Urbanek, A. 2014. "Hydrogen Recharge Dynamics and Vessel Design for Porous Silicon Storage Media." In Proceedings of TechConnect World 2014, National Harbor, Maryland.

[11] Schubert, P., Paganessi, J., Wilks, A., and Murray, M. 2012. "Distributed Hydrogen Generation and Storage from Biomass." In Materials Challenges in Alternative and Renewable Energy II, edited by G. Wicks, J. Simon et al. J. Wiley, Hoboken, New Jersey, 105-14.

[12] Witte, D., Kaushal, M., Jaithaliya, A., Mathur, A., and Schubert, P. 2013. "Scalable Hydrogen Production from Biomass." Proceedings Abstract of World Hydrogen Technologies Conference, Shanghai, China.

[13] Energy Research Centre of the Netherlands. 2016. Database for Biomass and Waste. Accessed 29 February 2016, https://www.ecn.n1/phyllis2/.

[14] Chemical Properties Handbook. 1999. McGraw-Hill Education. ISBN: 9780070734012.

[15] Huang, K., and Goodenough, J. B. 2009. Solid Oxide Fuel Cell Technology. Woodhead Publishing, CRC Press LLC.

[16] Lima, H., and Oyama, S. T. 2011. "Hydrogen Selective Thin Palladiumcopper Composite Membranes on Alumina Supports." J. Membr. Sci. 378 (1-2): 179-85. 
[17] Lehmann, V. 2002. Electrochemistry of Silicon: Instrumentation, Science, Materials, and Applications. Wiley-VCH.

[18] Tierney, H. L., Baber, A. E., Kitchin, J. R., and Sykes, E. C. H. 2009. "Hydrogen dissociation and Spillover on Individual Isolated Palladium Atoms." Phys Rev. Lett. 103: 46102.

[19] Schwalb, C., Lawrenz, M., Durr, M., and Hofer, U. 2007.
"Real-Space Investigation of Fast Diffusion of Hydrogen on $\mathrm{Si}(001)$ by a Combination of Nanosecond Laser Heating and STM.” Phys. Rev. B. 75: 085439.

[20] Weast, R. 1974. CRC Handbook of Chemistry and Physics (55th edition). CRC Press.

[21] DOE. 2016. Alternative Fuels Data Center. Accessed 1 March 2016 , http://www.afdc.energy.gov/fuels/prices.html. 